\title{
EEE
}

Homepage: http://publisher.uthm.edu.my/proceeding/index.php/eeee e-ISSN : 2756-8458

\section{Voltage Stability Analysis of Electric Power System with Integration of Renewable Energy}

\author{
Raudhah Izatee Ramlee ${ }^{1}$, Ahmad Fateh Mohamad Nor ${ }^{1,2 *}$ \\ ${ }^{1}$ Department of Electrical Engineering, \\ Faculty of Electrical and Electronics Engineering, \\ Universiti Tun Hussein Onn Malaysia, Batu Pahat, 86400, MALAYSIA \\ ${ }^{2}$ Green and Sustainable Energy Focus Group (GSEnergy), \\ Faculty of Electrical and Electronics Engineering, \\ Universiti Tun Hussein Onn Malaysia, Batu Pahat, 86400, MALAYSIA
}

DOI: https://doi.org/10.30880/eeee.2020.01.01.011

Received 10 August 2020; Accepted 06 September 2020; Available online 30 October 2020

\begin{abstract}
This study presents an analysis of voltage stability of the electrical power system with the integration of renewable energy and the improvement of the conventional analysis using the artificial neural network (ANN), which is mainly focused on the effect of distributed generator-solar (DG-Solar) towards the electric power system. Real power and reactive power margins of both voltage stability are expressed as VSM (P) and VSM (Q), respectively. They are obtained from the actual power-voltage $(\mathrm{PV})$ and reactive-power $(\mathrm{QV})$ curve, which is created for each series by a series of load flows by incrementing P and Q load. Then, the system will integrate with 5MW, 50MW and 100MW of DG-Solar to compare the electric power system before and after the integration. The results are observed and compared for both situations. After that, an analysis improvement is made by using ANN-based model to predict the values of VSM (P) and VSM (Q) without having to perform PV QV Curve and calculate VSM (P) and VSM (Q). IEEE 30-bus was chosen as the electrical power system. The load flow analysis and ANN-based model are simulated and developed by using MATLAB software.
\end{abstract}

Keywords: Voltage Stability Analysis, PV QV Curve, ANN, VSM (P), VSM (Q), DG-Solar

\section{Introduction}

In today's growth of modern society; the electrical infrastructure has become reliable and must use thriftily because the need for electricity has been increasing day by day. However, the non-renewable energy will run out and will not be replenished for thousands or even millions of years [1]. Besides, they give impact and damage to the environment and human health such as they will cause greenhouse gas and source of climate change which is carbon dioxide (CO2) [2]. In contrast, renewable energy sources produce much-cleaned energy and become attractive for many reasons [3]. However, renewable energy still gives impact towards voltage stability in an electric power system. 
Voltage stability is important to preserve the system's balance. It is about the ability of the electric power system to restore or sustain operating equilibrium and steady voltage at all busses under usual and abnormal conditions, after being subjected to disturbance and even after following increases of load [4][5]. In more significant words, it mainly occurs when the energy demand is greater than the network's capability [6]. In the form of context in this project, Distributed Generator-Renewable Energy will be attached to the power system, and the voltage stability is analyzed.

Evaluation of the voltage stability is a crucial parameter for controlling the bus voltage in the electric power system. Voltage instability occurs in the form pf a gradual decrease in voltages in some busses. This is due to the failure pf the electrical power grid to provide enough electricity to satisfy the growing load demand [7]. Thus, by installing the DG-Solar, it will give benefit toward the stability of the system.

This project will be focusing on comparing two (2) parts of results which are before and after the installation of DG-Solar at load buses in the power system in terms of its Voltage Stability. Distributed generation refers to a generation of electricity at or near where it will be used. It is becoming a new solution to improve the traditional network system [8]. The integration of the renewable distributed generator will enhance the system by improving the voltage profile and also by reducing power losses [9][10]. After that, Voltage Stability Margin (VSM) values will be analyzed by measuring the hypothenuse under the Real Power-Voltage (PV) and Reactive Power- Voltage (QV) curves [11]. VSM indicates how far the device will operate before voltage instability occurs. The smaller the VSM value, the closer the power system bus would be to voltage instability [12].

However, it takes many steps in obtaining the VSM values and both curves, which is difficult because whenever we want to obtain another result, the steps must be repeated from the initial step. To improve the conventional method of this analysis, Artificial Intelligence (AI) will be used, which is specified by using Artificial Neuron Network (ANN). By developing Artificial Neuron Network (ANN) based model on analyzing VSM values, this will be an advance and improved method to analyze the voltage stability analysis in the power system so the analysis can be done quickly without involving many steps.

The main aim of this study is to perform the voltage stability analysis on the IEE 30-bus electric power system while the sub-objective is comparing the VSM values under PV QV curve before and after the installation of DG-Solar. Next, ANN-based model is developed to predict the VSM values for the weakest bus and compare them with the manual calculated values from the generated PV QV curve conventional method analysis.

\section{Methodology}

The selected bus system that is simulated in this study is the IEEE 30-bus system, and all the data used are taken from the MATLAB file exchange. Moreover, a brief explanation about the PV QV curve method and calculation of VSM under the curves are explained also in this section. Last but not least, the usage of ANN in this study is defined in the last part of this section.

\subsection{System load modelling and data used}

Line diagram of the IEEE 30-bus system electrical power grid is shown in Figure 1. The network consists of one swing bus (Bus 1), 7 PV busses (Bus 2, Bus 4, Bus 5, Bus 8, Bus 10, Bus 11 and Bus 13), and 22 load busses (Bus 3, Bus 6, Bus 7, Bus 9, Bus 15 until Bus 30). The load flow analysis in this study is simulated using the MATLAB software and performed using the Gauss-Seidel power solution process while the coding used is taken from the MATLAB file exchange [13]. 


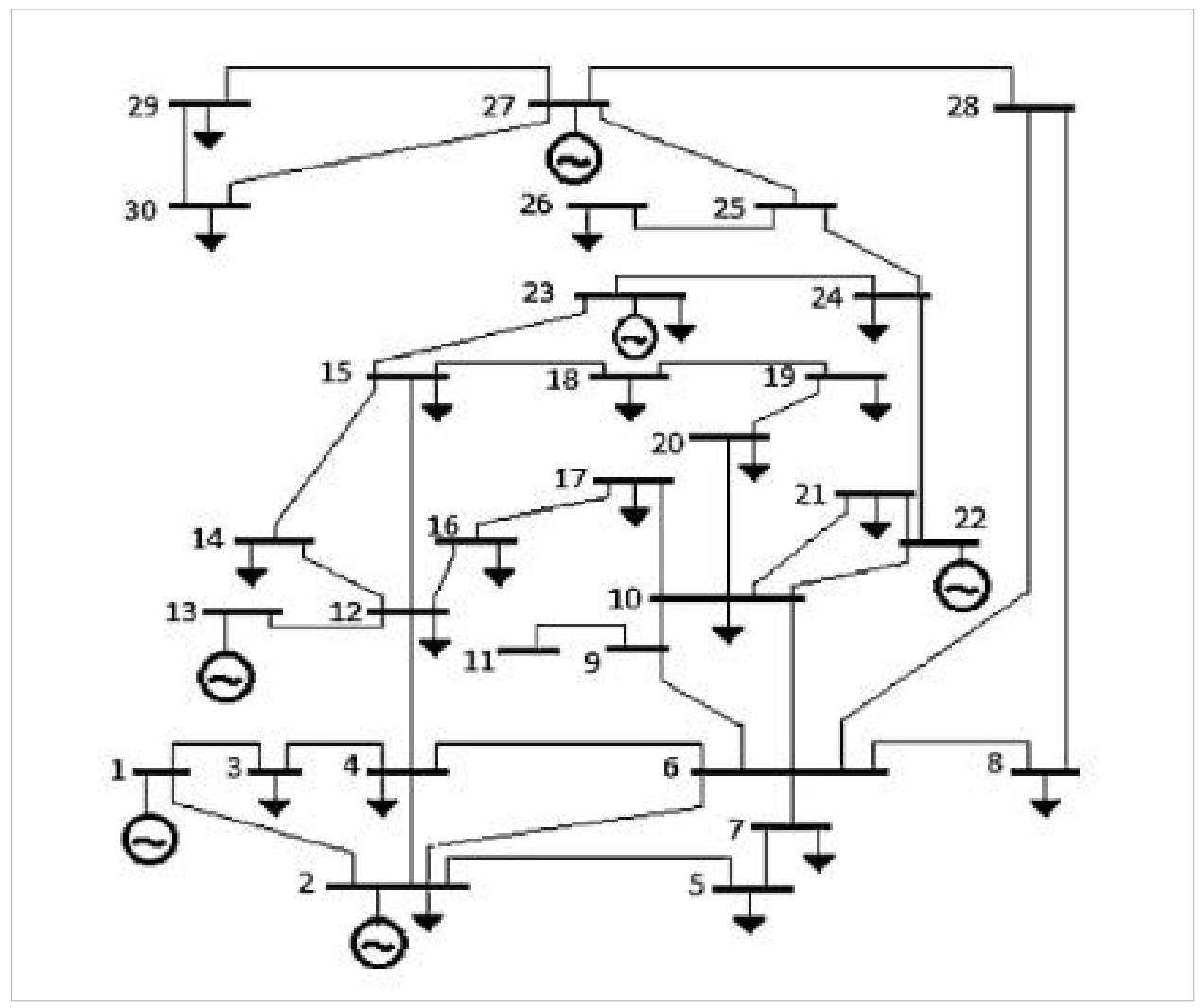

Figure 1: Line diagram of IEEE 30-bus electric power system [14]

\subsection{Obtaining VSM values under PV QV curve}

PV QV curve is very useful in the study of voltage stability. It produces a sequence of power flows. Load busses are the most vulnerable to voltage instability [15]. For each sequence of power flow, the system load is increased to the point that the system is no longer able to operate. The different values of $\mathrm{P}$ and $\mathrm{Q}$ with the load bus voltage are plotted as the PV QV curve. QV curve is the relationship between $\mathrm{Q}$ and receiving end voltage $(\mathrm{V})$ for different $\mathrm{P}$ values. Steps for lotting PV QV curves are as follows [11]:

- Run the series of load flow of IEEE 30-bus system by using MATLAB coding.

- The value of P load on the load bus is increased by 0.1 per unit for plotting PV curve. Similarly, the value of load $\mathrm{Q}$ is increased by 0.1 per unit for plotting the QV curve. After each increment, the load flow series will run again, and a new voltage value per unit is obtained.

- Repeat step (ii) until the incremented values of P and Q cannot be run anymore.

- Finally, by using the recorded values of P and Q obtained in the previous steps, plot them against bus voltage values.

Next, VSM can be defined as the distance between the initial operating voltage point and the critical voltage point [8]. VSM indicates how stable the system can be before voltage instability occurs. The lower the VSM values, the shorter the system will operate until it has voltage instability. VSM for actual power load (P) and VSM for reactive power load (Q) are referred to as VSM (P) and VSM (Q), respectively. VSM (P) is derived from the PV curve while the VSM (Q) is derived from the QV curve. Figure 2 shows the PV QV curve method to analyze the voltage stability of a system. 


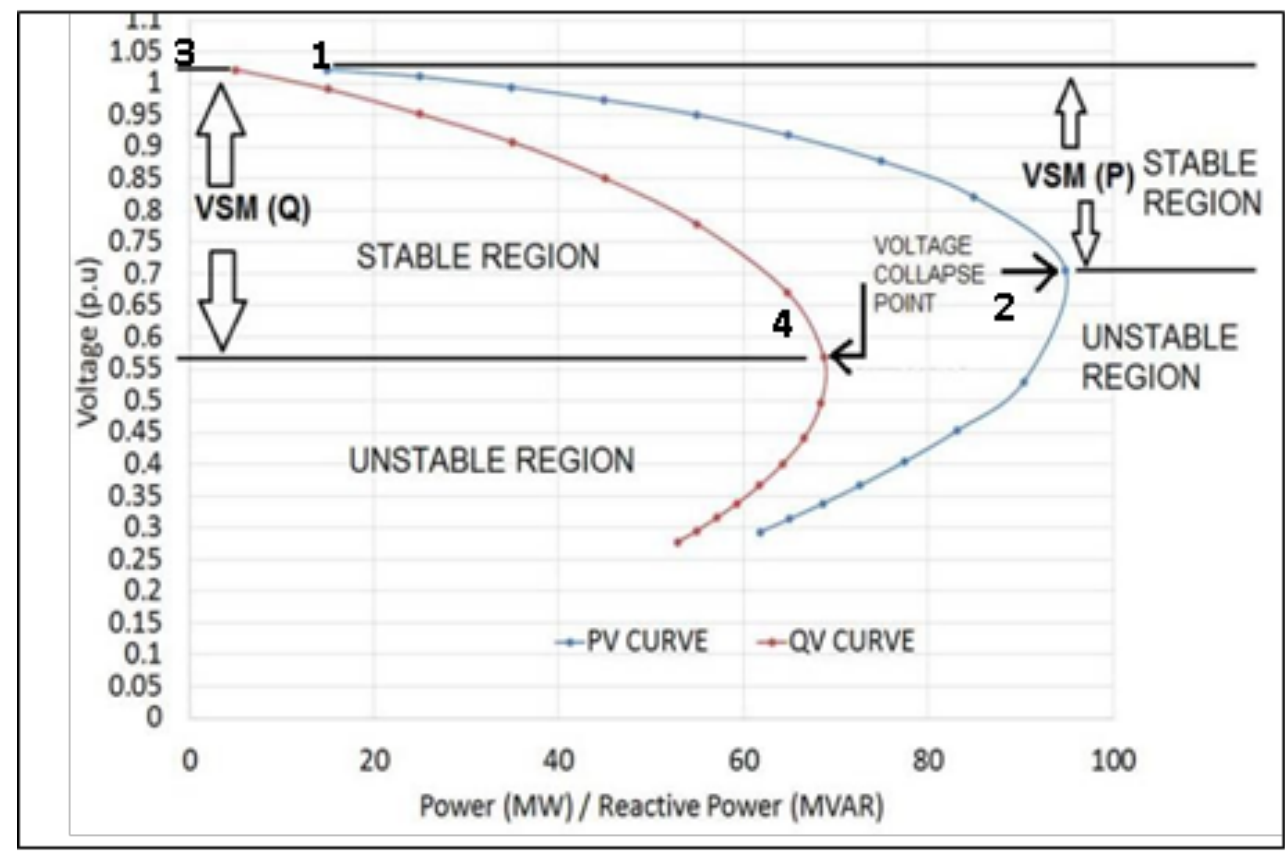

Figure 2: PV QV Curve [16]

According to Figure 2, let 1-4 as coordinates:

1 : ( $\left.P_{\text {initial }}, V_{\text {Pinitial }}\right)$

2 : ( $\left.P_{\text {critical }}, V_{\text {Pcritical }}\right)$

$3:\left(Q_{\text {initial }}, V_{\text {Qinitial }}\right)$

$4:\left(Q_{\text {critical }}, V_{Q c r i t i c a l}\right)$

$P_{\text {initial }}$ and $P_{\text {critical }}$ are the value of the real power of load voltage of the initial point and value of real power of load voltage at critical point, respectively. While $V_{\text {Pinitial }}$ and $V_{\text {Pcritical }}$ are the bus voltage at the initial and critical point, respectively. Thus, VSM (P) (Eq. 1) can be obtained by:

$$
\operatorname{VSM}(P)=\sqrt{\left(P_{\text {initial }}-P_{\text {critical }}\right)^{2}+\left(V_{\text {Pinitial }}-V_{\text {Pcritical }}\right)^{2}} \quad \text { Eq. } 1
$$

While $Q_{\text {initial }}$ and $Q_{\text {critical }}$ are the value of reactive power of load voltage at initial point and value of reactive power of load voltage at critical point, respectively. $V_{\text {Qinitial }}$ and $V_{\text {Qcritical }}$ are the bus voltage at the initial and critical points, respectively. Thus, VSM (Q) (Eq.2) can be calculated by:

$$
\operatorname{VSM}(Q)=\sqrt{\left(Q_{\text {initial }}-Q_{\text {critical }}\right)^{2}+\left(V_{\text {Qinitial }}-V_{\text {Qcritical }}\right)^{2}} \quad \text { Eq. } 2
$$

\subsection{Developing ANN-based model prediction}

In the conventional analysis, the PV QV curve and VSM calculation must be plotted and calculated manually. The ANN-based model must be trained developed and used to predict VSM values. In order to do this, the ANN-based model must be trained to learn the cycle between the input quantity and output. Next, Mean Squared Error (MSE) analysis is used to denote the efficiency of the ANN-based model. MSE is the mean squared difference between output and goals. A higher value indicates a higher error, so zero indicates no error. Thus, the closer the MSE values to zero, the better the performance of the ANN-based model and its prediction. MSE analysis is calculated as Eq. 3 [17]:

$$
M S E=\frac{1}{N T D} \sum_{T D=1}^{N T D}\left((\text { Target }-A N N \text { Prediction })^{2}\right) \quad \text { Eq. } 3
$$


: The total number of training data

: The number of training data

By developing ANN-based model, $V_{\text {initial }}, V_{\text {critical }}$, and size pf DG-Solar will be the input data while the target data is the actual VSM (P) or VSM (Q) data. In addition, steps in developing ANNbased model for VSM predicition are including:

- Insert input data ( $V_{\text {initial }}, V_{\text {critical }}$ and size od DG-Solar) to represent the input variable in the network.

- Insert target data (actual VSM values) to represent the desired network output.

- Train all the input and target variables.

- Produce graph of MSE to indicate the performance of the VSM prediction.

- Record the output and error from the ANN-based model VSM predicted values.

With 200 training data used in this ANN-based model, they are divided into 3 types of data samples which are $70 \%$ of training data, $15 \%$ of validation data, and $15 \%$ of testing data. They are 140 samples, 30 samples, and 30 samples respectively. Meanwhile, the hidden layer contains 10 numbers of neutrons. The division of data sample and hidden neutron is according to ANN MATLAB default.

\section{Results and Discussion}

The results that have been obtained throughout this study are analyzed and explained in this section. The electric power system of the IEEE 30-bus system is analyzed using the conventional method, which is by calculating VSM (P) and VSM (Q) values under the PV QV curve method. After that, the analysis improvement of the conventional method is performed by using the ANN-based model in MATLAB software.

\subsection{Load flow analysis of IEEE-30 bus electric power system}

In this section, load flow analysis is introduced for the acquisition of voltage, active and reactive power at each power system bus to analyze its steady-state conditions. Then, per load flow series, the system loads are increased to the point that the system is no longer able to operate. The real power $(\mathrm{P})$ and reactive power $(\mathrm{Q})$ variance load values with the bus voltage values are plotted as the PV QV curve. After that, VSM (P) and VSM (Q) are calculated under PV and QV curve, respectively. Table 1 shows the value of VSM (P) and VSM (Q) for each load busses.

According to Table 1, Bus 26, Bus 27 and Bus 30 have the same value of VSM (P) and VSM (Q). Thus, in order to differentiate them, the difference in their curves are analysed. They took the same amount of iteration before they experience voltage instability which is three (3) iterations. But even though they have the same amount of iterations, Bus 27 will be the weakest because its P and Q load starts with zero. Moreover, Bus 27 has a smaller curve compared to Bus 26 and Bus 30 which indicate that they have a higher incremental of load P and Q before they are experiencing voltage instability.

Table 1: Values of VSM (P) and VSM (Q)

\begin{tabular}{ccc}
\hline Load Buses & $\begin{array}{r}\text { VSM (P) } \\
\text { (per unit) }\end{array}$ & $\begin{array}{c}\text { VSM (Q) } \\
\text { (per unit) }\end{array}$ \\
\hline \hline 3 & 1.0 & 0.5 \\
6 & 0.4 & 0.4 \\
7 & 0.4 & 0.2 \\
9 & 0.4 & 0.4 \\
12 & 0.5 & 0.4 \\
14 & 0.4 & 0.3 \\
15 & 0.4 & 0.3 \\
\hline 16 & 0.4 & 0.4
\end{tabular}




\begin{tabular}{lll}
17 & 0.4 & 0.4 \\
18 & 0.4 & 0.3 \\
19 & 0.4 & 0.3 \\
20 & 0.4 & 0.3 \\
21 & 0.4 & 0.4 \\
22 & 0.5 & 0.4 \\
23 & 0.4 & 0.3 \\
24 & 0.4 & 0.3 \\
25 & 0.3 & 0.3 \\
26 & 0.2 & 0.2 \\
27 & 0.2 & 0.2 \\
28 & 0.5 & 0.2 \\
29 & 0.3 & 0.2 \\
30 & 0.2 & 0.2 \\
\hline
\end{tabular}

3.2 IEEE 30-bus system with integration of DG-solar

Since the load bus 27 is the weakest, it became the chosen bus to integrate with DG-Solar since this bus is the closest towards voltage instability. DG-Solar that is installed at load bus 27 is divided into three conditions which are DG-Solar with 5MW, DG-Solar with 50MW, and DG-Solar with 100MW. The effects of these installations are observed in PV QV curve as in Figure 3, Figure 4 and Figure 5. Thus, the larger the size of DG-solar, the larger the size of the voltage stability margin.

From PV QV curve for Bus 27 after the installation of 5MW DG-solar in Figure 3, it shows that the increment of the $\mathrm{P}$ and $\mathrm{Q}$ load only can reach maximum up to $40 \mathrm{MW}(0.4$ per unit) and 30MVar (0.3 per unit), respectively. While Figure 4 shows the PV QV curve for Bus 27 after the 50MW DG Solar installation. The P load can withstand up to $70 \mathrm{MW}$ with 0.7 per unit of load $\mathrm{P}$ increment while its $\mathrm{Q}$ load can withstand until 50MVar before experiencing voltage instability. As shown in Figure 6, Bus 27 with 100MW DG-Solar is able to reach 120MW (1.2 per unit) and 40MVar (0.4 per unit) before the system is collapses and not stable.

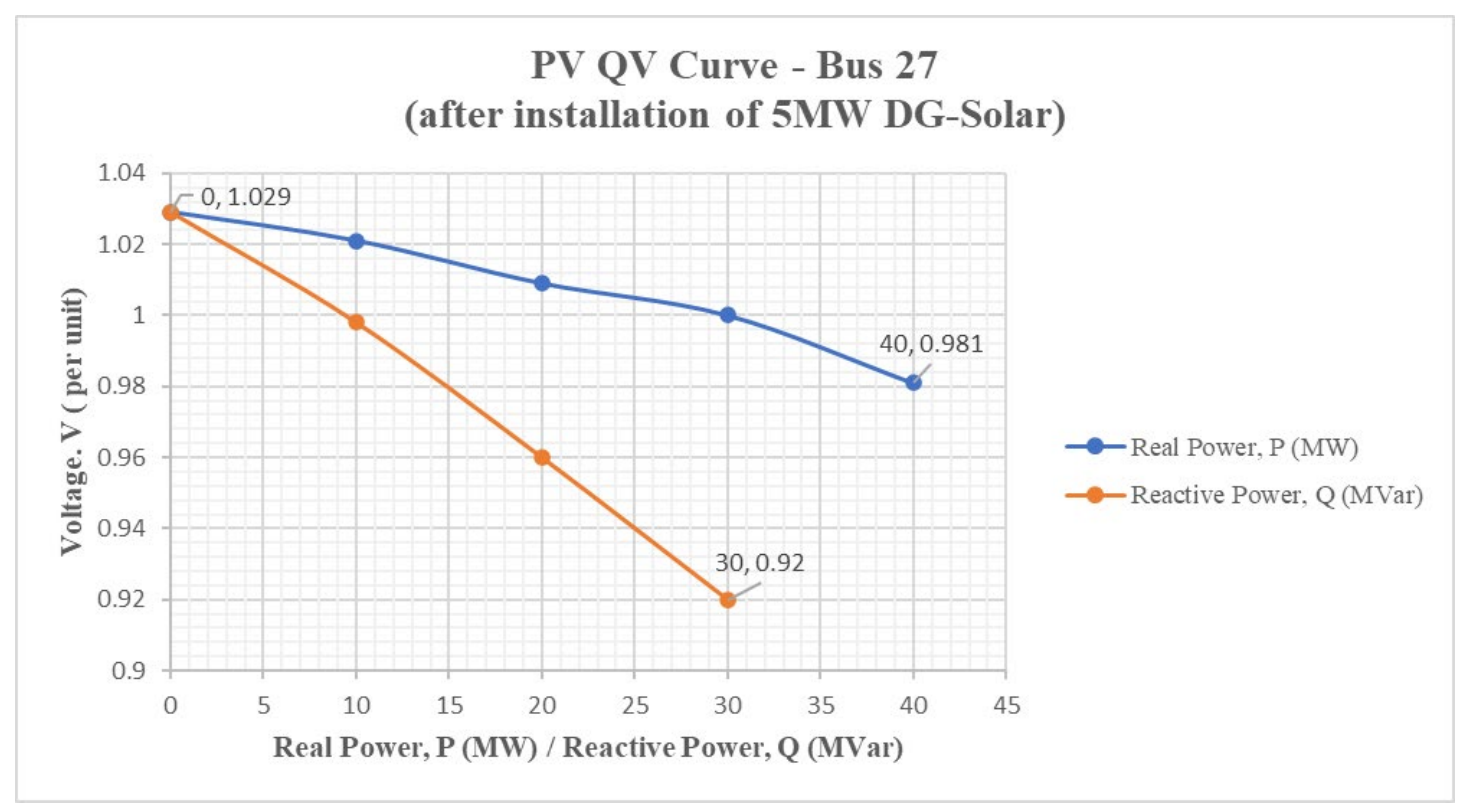

Figure 3: PV QV curve for Bus 27 with 5MW DG-solar 


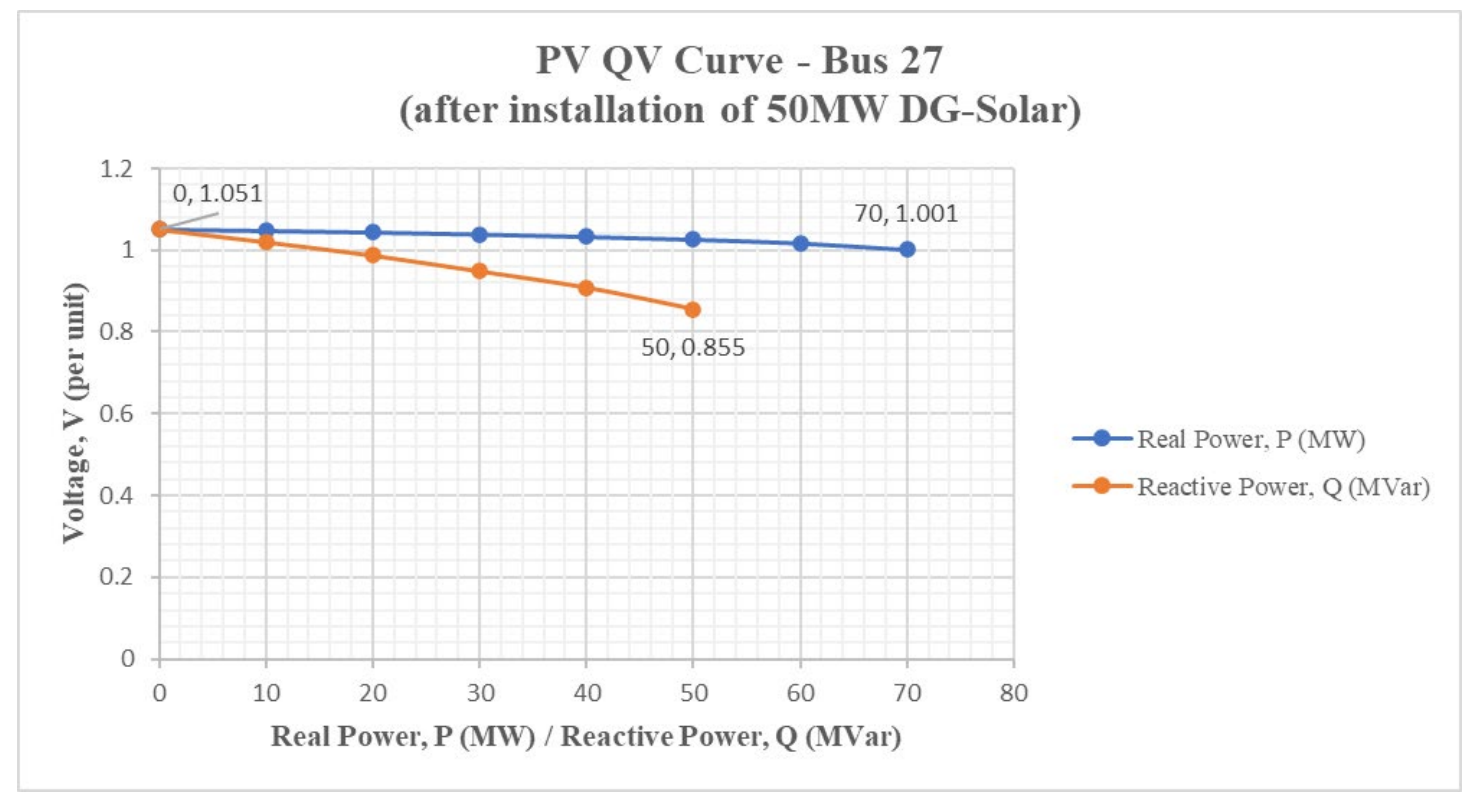

Figure 4: PV QV curve for Bus 27 with 50MW DG-solar

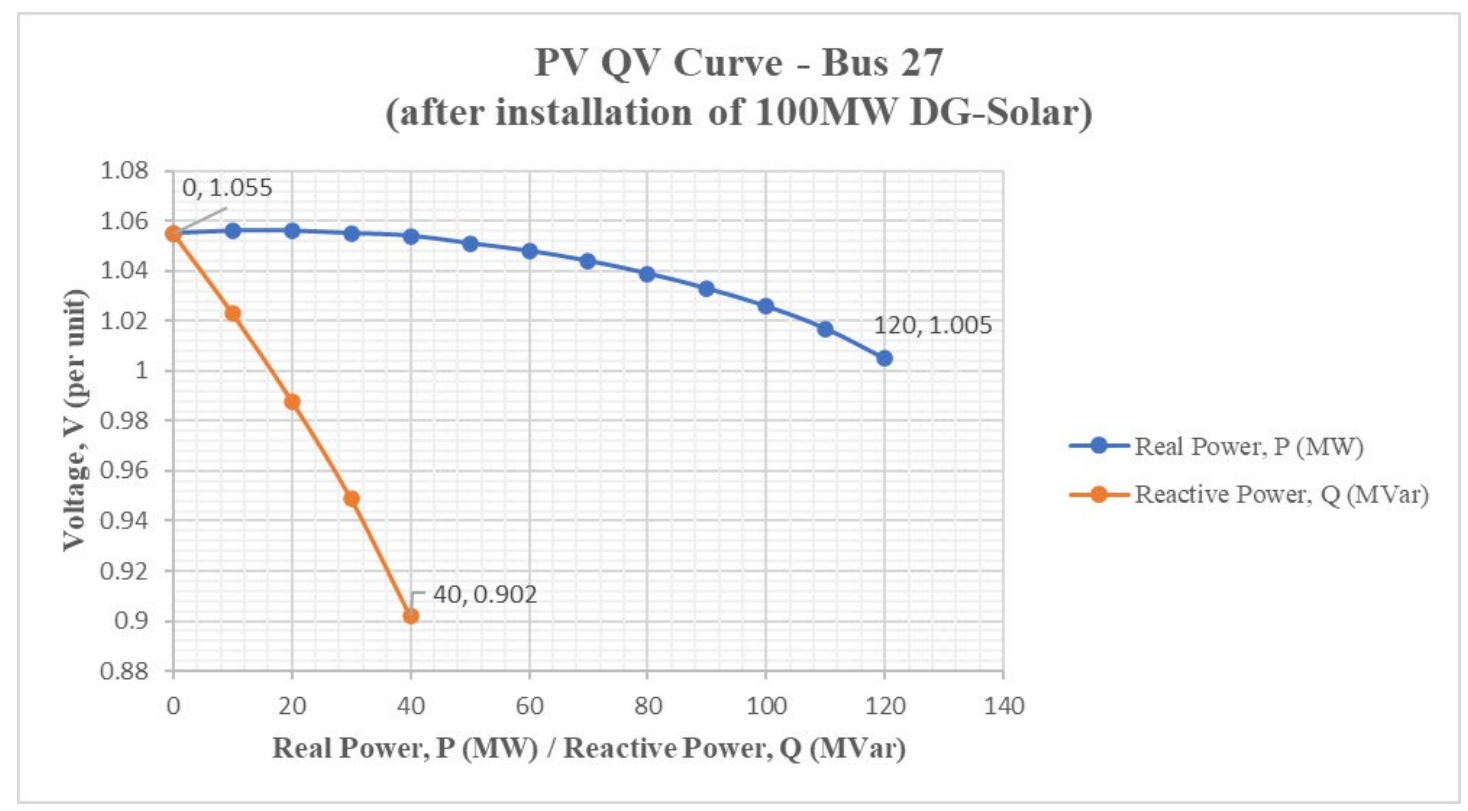

Figure 5: PV QV curve for Bus 27 with 100MW DG-solar

Table 2 below shows the comparison of the values of VSM (P) and VSM (Q) on each condition towards Bus 27. Load Bus 27 with 5MW DG-Solar has a higher value of VSM compared to load Bus 27 without DG-Solar. It shows that by installing DG-Solar to the weakest bus in the system, it will improves the VSM values.

Table 2: Values of VSM (P) and VSM (Q) at load bus 27

\begin{tabular}{ccc}
\hline Load Bus 27 & $\begin{array}{c}\text { VSM (P) } \\
\text { (per unit) }\end{array}$ & $\begin{array}{c}\text { VSM (Q) } \\
\text { (per unit) }\end{array}$ \\
\hline \hline Without DG-Solar & 0.2 & 0.2 \\
With 5MW DG-Solar & 0.4 & 0.3 \\
With 50MW DG-Solar & 0.7 & 0.5 \\
With 100MW DG-Solar & 1.2 & 0.4 \\
\hline
\end{tabular}


Thus, through these results, it can be confirmed that by placing a DG-Solar, it can improves the VSM (P) values since the integration of DG-Solar only involves the addition of real power, P. Even though there is an increasing value of VSM (P), but for overall voltage stability to improve, the primary concern is the value of VSM $(\mathrm{Q})$. But in this study, the integration of 100MW DG-Solar causing lower voltage stability compared to 50MW DG-Solar which is 0.5 per unit. It means that 50MW DG-Solar has a greater optimal size compared to 100MW DG-Solar.

All in all, if Bus 27 without DG-Solar and Bus 27 with 5MW DG-Solar are compared, the integration of DG-Solar to the weakest bus in the system still improves the value of VSM. Since the size of DG-Solar is set randomly in this study, 100MW DG-Solar is found to not have optimal sizing for this system compared to 50MW DG-Solar.

\subsection{Improvement analysis using ANN}

By developing the ANN-based model, it can be used to predict the values of VSM without having to involve the plotting and calculating parts. The ANN-based model itself will predict the values of VSM (P) and VSM (Q) and the error from the prediction analysis will also be given from the MATLAB. Table 3 shows the comparison between actual and ANN predicted VSM (P) values and its percentage of error as well. While Figure 6 shows the graph of MSE produced in the VSM (P) ANN-based model prediction.

Table 3: Comparison of VSM (P) results and its error obtained

\begin{tabular}{ccccc}
\hline & \multicolumn{4}{c}{ VSM (P) } \\
\cline { 2 - 5 } Load Bus 27 & $\begin{array}{c}\text { Actual } \\
\text { (per unit) }\end{array}$ & $\begin{array}{c}\text { ANN Prediction } \\
\text { (per unit) }\end{array}$ & Error & Error (\%) \\
\hline \hline Without DG-Solar & 0.2 & 0.2 & $3.243 \times 10^{-12}$ & $3.243 \times 10^{-10}$ \\
With 5MW DG-Solar & 0.4 & 0.4 & $3.927 \times 10^{-10}$ & $3.927 \times 10^{-8}$ \\
With 50MW DG-Solar & 0.7 & 0.7 & $5.851 \times 10^{-10}$ & $5.851 \times 10^{-8}$ \\
With 100MW DG-Solar & 1.2 & 1.2 & $1.345 \times 10^{-9}$ & $1.345 \times 10^{-7}$ \\
\hline
\end{tabular}

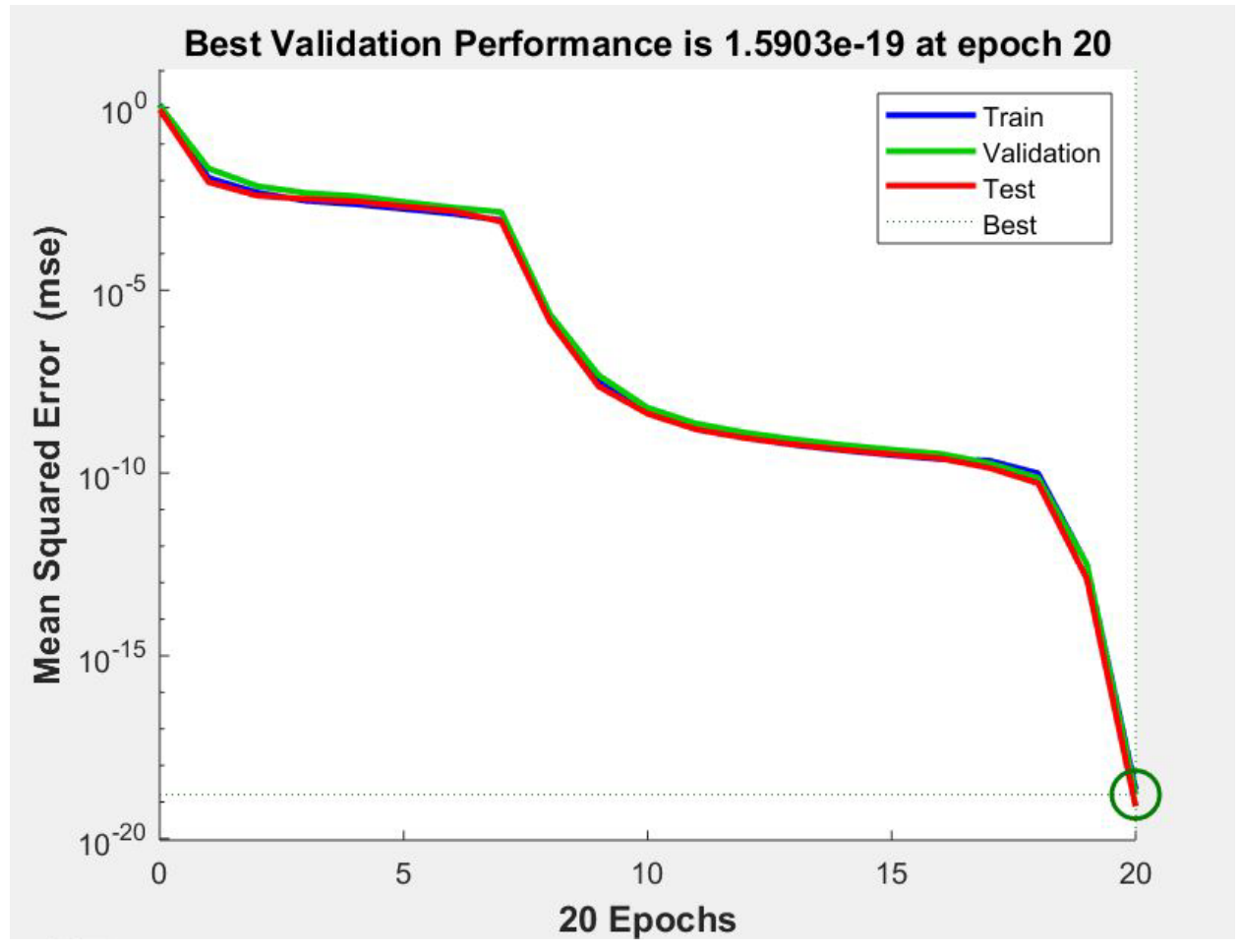

Figure 6: Graph of MSE performance on VSM (P) prediction 
From Table 3, it shows that only a small difference in value between actual and ANN predicted VSM (P) values. The highest percentage error in this prediction is only $1.345 \times 10^{-7} \%$ while the lowest percentage error is $3.243 \times 10^{-10 \%}$. Besides, according to Figure 6, MSE for this ANN-based model for VSM (P) prediction is only $1.5903 \times 10^{-19}$ with 20 epochs on average to perform the best validation performance. Since the value is very close to zero, it indicates the best performance for the ANN-based model for VSM (P) prediction. Next, Table 4 shows the comparison between actual and ANN predicted values for VSM (Q) as well as its error obtained. Meanwhile, Figure 7 shows the graph of MSE produced for VSM (Q) ANN-based model prediction.

Table 4: Comparison of VSM $(Q)$ results and its error obtained

\begin{tabular}{ccccc}
\hline & \multicolumn{4}{c}{ VSM (Q) } \\
\cline { 2 - 5 } Load Bus 27 & $\begin{array}{c}\text { Actual } \\
\text { (per unit) }\end{array}$ & $\begin{array}{c}\text { ANN Prediction } \\
\text { (per unit) }\end{array}$ & Error & Error (\%) \\
\hline Without DG-Solar & 0.2 & 0.1999 & $1.557 \times 10^{-10}$ & $1.557 \times 10^{-8}$ \\
With 5MW DG-Solar & 0.3 & 0.2999 & $3.221 \times 10^{-10}$ & $3.221 \times 10^{-8}$ \\
With 50MW DG-Solar & 0.5 & 0.4999 & $1.567 \times 10^{-10}$ & $1.567 \times 10^{-8}$ \\
With 100MW DG-Solar & 0.4 & 0.4 & $5.508 \times 10^{-10}$ & $5.508 \times 10^{-8}$ \\
\hline
\end{tabular}

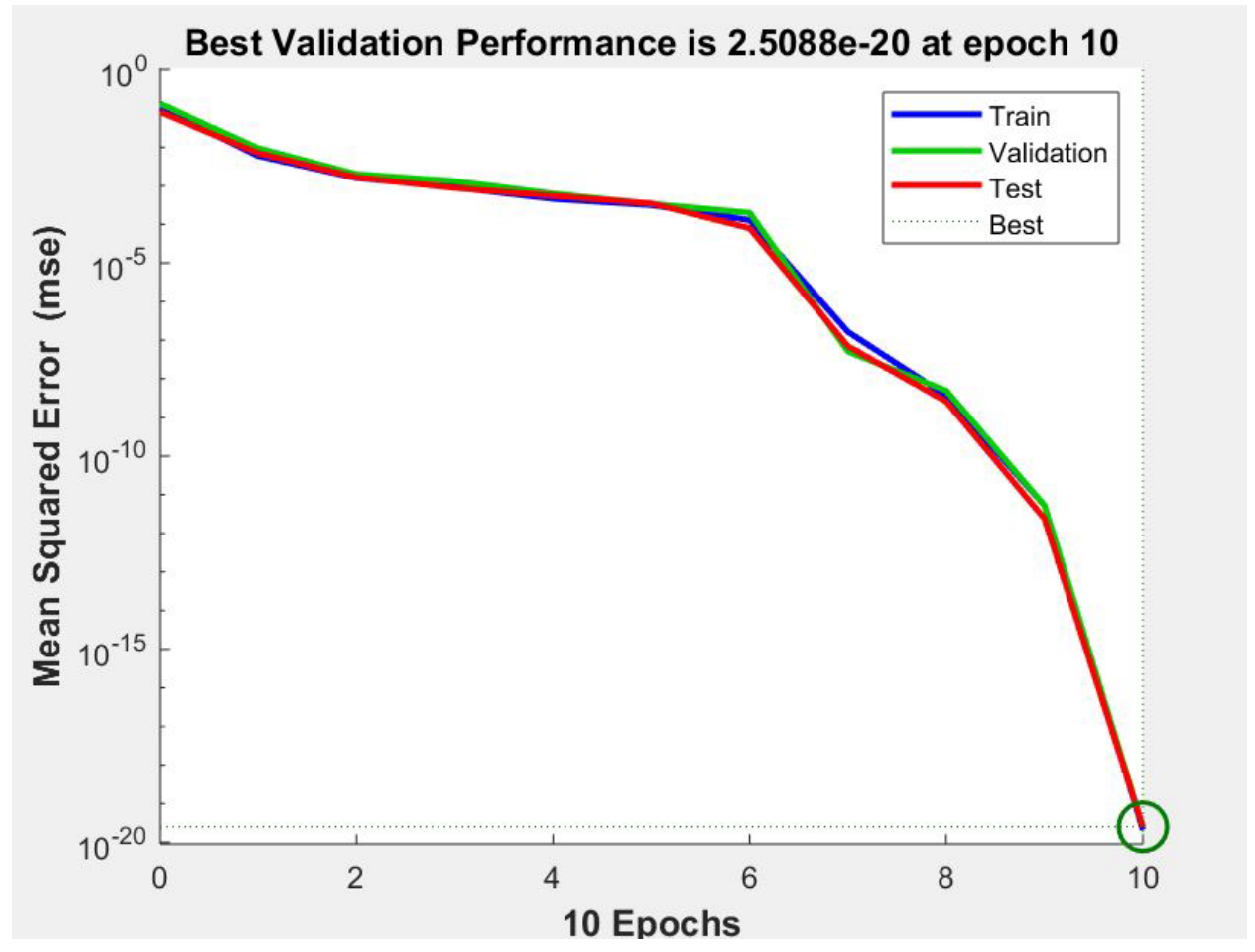

Figure 7: Graph of MSE performance on VSM (Q) prediction

Meanwhile, Table 4 shows that only a small difference in value between actual and ANN predicted VSM (Q) values. With 10 epochs, the highest percentage error in this VSM (Q) prediction is 5.508x10$8 \%$ while the lowest percentage error is $1.557 \times 10^{-8} \%$ and it can be considered negligible. Besides, according to Figure 7, it shows the MSE performance of this ANN-based model for VSM (Q) prediction is only $2.5088 \times 10^{-20}$ with 10 epochs on average to perform its almost accurate validation performance. Since the value is very close to zero. In instance, error obtained from the ANN prediction of both VSM $(\mathrm{P})$ and VSM (Q) can be neglected. 


\section{Conclusion}

The study conducted in this paper show that voltage stability of an electric power system can be analyzed through observing PV QV curve and its VSM values. Not only that, this study also proves that DG-Solar can give positive impact towards the voltage stability of an electric power system which they can improve the value of VSM . Last but not least, ANN-basd model that is developed in MATLAB can be useful because of its prediction that can be initial precaution to avoid voltage collapse or instability.

\section{Acknowledgement}

The authors would like to thank to the Faculty of Electric and Electronic Engineering, Universiti Tun Hussein Onn Malaysia for its support.

\section{References}

[1] M. Hasani and M. Parniani, "Method of combined static and dynamic analysis of voltage collapse in voltage stability assessment," Proc. IEEE Power Eng. Soc. Transm. Distrib. Conf., vol. 2005, pp. 1-6, 2005

[2] Y. Shao and Y. Tang, "Voltage stability analysis of multi-infeed HVDC systems using smallsignal stability assessment," 2010 IEEE PES Transm. Distrib. Conf. Expo. Smart Solut. a Chang. World, pp. 1-6, 2010

[3] M. R. Islam, M. Numan, M. Shahzad, M. M. Islam, and M. M. Rana, "Voltage and transient stability analysis in Bangladesh's power system network (BPSN)," Proc. 2017 12th IEEE Conf. Ind. Electron. Appl. ICIEA 2017, vol. 2018-Febru, pp. 866-870, 2018

[4] Z. Chen et al., "Voltage stability analysis of wind farms integration connected to distribution network," 2017 IEEE Conf. Energy Internet Energy Syst. Integr. EI2 2017 - Proc., vol. 2018Janua, pp. 1-5, 2018

[5] W. Suampun, "Voltage Stability Analysis of Grid-connected Photovoltaic Power Systems Using CPFLOW,” Procedia Comput. Sci., vol. 86, no. March, pp. 301-304, 2016

[6] "Learn more about Voltage Stability Derivation of Distribution Network Vulnerability Indicators Based on Volt- age Stability Stability problems of distributed gener- ators," 2019

[7] "Distributed Generation of Electricity and its Environmental Impacts | Energy and the Environment | US EPA." [Online]. Available: https://www.epa.gov/energy/distributedgeneration-electricity-and-its-environmental-impacts. [Accessed: 20-Jul-2020]

[8] M. T. Turan, Y. Ates, O. Erdinc, and E. Gokalp, "Effect of Distributed Generation Based Campus Model Combined with Electric Vehicle Charging Stations on the Distribution Network,” SEST 2019 - 2nd Int. Conf. Smart Energy Syst. Technol., 2019

[9] N. Dhlamini and S. P. Daniel Chowdhury, "Solar Photovoltaic Generation and its Integration Impact on the Existing Power Grid," 2018 IEEE PES/IAS PowerAfrica, PowerAfrica 2018, pp. $710-715,2018$

[10] A. M. ELTAMALY, Y. S. MOHAMED, A.-H. M. EL-SAYED, and A. N. A. ELGHAFFAR, "Impact of Distributed Generation (Dg) on the Distribution System Network.," Ann. Fac. Eng. Hunedoara - Int. J. Eng., vol. 17, no. 1, pp. 165-170, 2019

[11] A. Ghosh, R. Patel, M. Datta, and L. Meegahapola, "Investigation of transient stability of a 
power network with solar-PV generation: Impact of loading level \& control strategy," 2017 IEEE Innov. Smart Grid Technol. - Asia Smart Grid Smart Community, ISGT-Asia 2017, pp. $1-6,2018$

[12] "Distributed Solar Power Energy - Fourth Partner Energy." [Online]. Available: https://fourthpartnerenergy.wordpress.com/category/distributed-solar-power-energy/.

[Accessed: 20-Jul-2020]

[13] Souhardya Panda, "Load Flow analysis of 6-bus, 9-bus, 14-bus, 26-bus \& 30-bus test system by GS, NR and fast decoupled - File Exchange - MATLAB Central," MathWorks, 2017. [Online]. Available: https://www.mathworks.com/matlabcentral/fileexchange/65077-load-flow-analysisof-6-bus-9-bus-14-bus-26-bus-30-bus-test-system-by-gs-nr-and-fast-decoupled. [Accessed: 21Jul-2020]

[14] P. K. Hota and A. P. Naik, "Least loss contract in deregulated power system using relative electrical distance (RED) concept,” J. Electr. Syst. Inf. Technol., vol. 5, no. 3, pp. 502-525, 2018

[15] U. Princy, S. Jaseena, S. Sreedharan, and S. Sreejith, "Voltage stability analysis of power system network integrated with renewable source and SVC," 2017 Innov. Power Adv. Comput. Technol. i-PACT 2017, vol. 2017-Janua, pp. 1-6, 2017

[16] A. F. M. Nor, M. Sulaiman, and R. Omar, "Study of voltage and power stability margins of electrical power system using ANN," IET Conf. Publ., vol. 2016, no. CP688, pp. 7-13, 2016

[17] A. R. Bahmanyar and A. Karami, "Power system voltage stability monitoring using artificial neural networks with a reduced set of inputs," Int. J. Electr. Power Energy Syst., vol. 58, pp. 246-256, 2014 